\title{
Determinación del colesterol en pacientes ingresados por eventos coronarios agudos y prevención secundaria de la cardiopatía isquémica
}

\author{
J. A. GARRIDO SANJUÁN, G. PÍA IGLESIAS, P. SESMA SÁNCHEZ* \\ Unidad de Lípidos. Servicio de Medicina Interna. Hospital Arquitecto Marcide. Ferrol. \\ *Departamento de Medicina. Universidad de Santiago. Santiago de Compostela. \\ La Coruña
}

SERUM CHOLESTEROL DURING THE COURSE ACUTE CORO -
NARY EVENTS AND SECONDARY PREVENTION OF CORONARY HEART DISEASE

\section{RESUMEN}

Objetivos: Evaluar las modificaciones de la concentración plasmática de colesterol (CT) durante los eventos coronarios agudos (infarto -IAM- y angina inestable) en las condiciones de la práctica clínica, con objeto de plantearse el inicio de tratamiento farmacológico hipolipemiante precoz con una sola determinación lipídica conocida.

Métodos: Revisión de la base de datos de la Unidad de Lípidos de un hospital de 400 camas. Criterio de selección: pacientes enviados a la Unidad entre uno y tres meses después del evento coronario agudo, que no hubieran iniciado tratamiento hipolipemiante farmacológico.

Resultados: 56 pacientes, $69 \%$ con IAM. La media de CT durante el ingreso fue menor. Dieciseis de los 17 con CT menor de $200 \mathrm{mg} / \mathrm{dl}$ en fase aguda tenían CT mayor de 200 en la consulta. El mayor descenso en fase aguda se produjo entre aquellos con CT más elevada en consulta.

Conclusiones: Se confirma el descenso de la CT en la fase aguda del IAM y se describe su descenso también en la angina inestable. Estos resultados permiten plantear en la mayoría de las ocasiones un inicio precoz del tratamiento farmacológico hipolipemiante, aún siendo la única determinación lipídica conocida.

PALABRAS CLAVE: Prevención secundaria de cardiopatía isquémica. Colesterol. Lípidos,. Fármacos hipolipemiantes. Estatinas. Infarto agudo de miocardio. Angina inestable.

\begin{abstract}
Objectives: To asess changes in the serum cholesterol (CT) during acute coronary events (infarction-IAM-and unstable angina) in clinical practice, to raise to begin pharmacologic lipid therapy knowing only a lipid profile.

Methods: Lipid clinic database was revised. Selection criteria: patients who were sent to the clinic 1 to 3 months after the acute coro nary event and who were not begun pharmacologic lipid therapy.

Results: 56 pacients, 69\% with IAM. Admission CT mean was lower. Among 17 who had CT lower than $200 \mathrm{mg} / \mathrm{dl}$ during acute phase, $16 \mathrm{had}$ CT higher than 200 in ambulatory lipid profile. The bigger acute phase drop took place between those who had CT higher during ambulatory consultation.

Conclusions: Theses results confirm the CT decrease during IAM and reports this reduction happens during unstable angina too. That per mits to suggest mostly to begin the lipid lowering drug therapy earlier, even although there was only a lipid vlue known.
\end{abstract}

KEY WORDS: Coronary heart disease secondary prevention. Choleste rol. Lipids. Lipid lowering drug therapy. Statins. Acute myocardial infarct. Unstable angina.

Garrido Sanjuán JA, Pía Iglesias G, Sesma Sánchez P. Determinación del colesterol en pacientes ingresados por eventos coronarios agudos y prevención secundaria de la cardiopatía isquémica. An Med Interna (Madrid) 2001; 18: 464-467.

\section{INTRODUCCIÓN}

El momento en que se debe iniciar el tratamiento hipolipemiante en pacientes que han tenido un infarto agudo de miocardio (IAM) no ha sido todavía claramente definido. Los consensos más asentados en la práctica clínica están redactados antes de la consolidación del beneficio de las estatinas en la prevención secundaria $(1,2)$. Existe clásicamente la idea de que la colesterolemia no deja de estar falsamente disminuida hasta 2 o 3 meses después del IAM $(3,4)$. Sin embargo los descensos más importantes se producen entre los días 4 y 12 , con descensos porcentuales de 24 al
$70 \%$ respecto a los valores basales (3), y hay varios trabajos en que las concentraciones de colesterol regresan a sus valores basales a las 3 o 4 semanas $(3,5)$. La mayoría de los trabajos que han permitido el conocimiento del descenso de la colesterolemia en fase aguda del infarto de miocardio están realizados en condiciones de "observación experimental", con determinaciones realizadas en períodos exactos prefijados después del inicio del evento agudo, e incluyen un número reducido de pacientes: 22 y 58 pacientes en los trabajos reiteradamente citados de Tibblin y Cramer (5) y Ryder et al. (6), 83 pacientes en el estudio procedente de la cohorte de Framingham (7), etc.

Trabajo aceptado: 27 de marzo de 2001

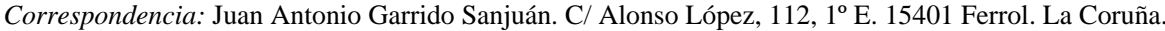


Por otro lado, como norma generalmente aceptada no se debe iniciar tratamiento farmacológico hipolipemiante sin constatar la elevación de las concentraciones de colesterol y/o triglicéridos en al menos dos determinaciones separadas por dos o tres semanas (8). Sin embargo las nuevas evidencias que muestran el beneficio de las estatinas en la prevención secundaria de la cardiopatía isquémica podrían establecer una excepción a esta regla general, si se reafirma en la práctica clínica que el falso descenso de la colesterolemia que se produce en la fase aguda permite presuponer concentraciones de colesterol que están realmente por encima de las concentraciones que implican inicio de tratamiento farmacológico.

Tiene interés, por tanto, la evaluación de lo que ocurre con estas cifras en las condiciones determinadas por la práctica clínica. En el presente trabajo hemos evaluado si existían datos para sustentar el inicio de tratamiento farmacológico con una sola determinación lipídica conocida en pacientes con IAM y concentraciones de colesterol "normales" o moderadamente elevadas, en nuestro centro. Asimismo el trabajo incluye la valoración en el mismo sentido de casos de angina inestable, sin evidencia de necrosis, datos que es difícil encontrar en la literatura.

\section{MATERIAL Y MÉTODOS}

Se realizó una revisión de la base de datos de la Unidad de Lípidos correspondiente a un hospital de 400 camas que atiende un área sanitaria de 220.000 habitantes. Se seleccionaron aquellos pacientes que habían sido enviados a la Unidad entre 1 y 3 meses después de un ingreso por un evento coronario agudo (IAM o angina inestable), sin haber iniciado tratamiento farmacológico hipolipemiante al alta hospitalaria. Se compararon las concentraciones de lípidos realizadas durante el ingreso, que por la práctica habitual en el centro están realizadas al menos 24 horas y generalmente más de 48 horas después del ingreso, con las obtenidas en la primera consulta en la Unidad, mediante test de "t de Student" para datos pareados. La concentración de colesterol de lipoproteínas de baja densidad (cLDL) se calculó mediante la fórmula de Friedwald en aquellos con triglicéridos menores de $400 \mathrm{mg} / \mathrm{dl}$. El método enzimático para la determinación de colesterol y triglicéridos, mediante autoanalizador, fue el mismo en ambas determinaciones. Siendo un estudio retrospectivo, sólo tenían determinación del perfil lipídico completo en ambas extracciones 18 pacientes.

\section{RESULTADOS}

Cincuenta y seis pacientes, 45 varones y 11 mujeres, cumplían el criterio de selección. Su edad media era de 59,2 años, con rango de 30 a 81 años. Habían presentado infarto $38(67,8 \%)$. La concentración de colesterol total durante el ingreso fue significativamente menor que en la determinación posterior ambulatoria (Tabla I). El 78,5\% (44 pacientes) presentaron colesterolemia menor en fase aguda, 31 con IAM y 13 de los 18 con angor. Dieciséis de los 17 con colesterol menor de $200 \mathrm{mg} / \mathrm{dl}$ en fase aguda presentaron colesterol mayor de dicha concentración en consulta. Dieciocho pacientes tenían determinación de cHDL en fase aguda además de en consulta. La concentración de cLDL calculada en

\begin{tabular}{|c|c|c|c|c|}
\hline \multicolumn{5}{|c|}{ TABLA I } \\
\hline Determinación & Pacientes & $\begin{array}{c}\text { Fase aguda } \\
\text { media (DE) } \\
\text { rango }\end{array}$ & $\begin{array}{l}\text { Consulta } \\
\text { media (DE) } \\
\text { rango }\end{array}$ & $p$ \\
\hline \multirow[t]{3}{*}{$\begin{array}{l}\text { Colesterol total } \\
(\mathrm{mg} / \mathrm{dl})\end{array}$} & Todos (56) & $\begin{array}{c}222(38) \\
151-301\end{array}$ & $\begin{array}{c}251(36) \\
179-417\end{array}$ & $p<0,001$ \\
\hline & IAM (38) & $218(34)$ & $249(41)$ & $p<0,001$ \\
\hline & Angor (18) & $234(45)$ & $254(26)$ & $p=0,054$ \\
\hline Triglicéridos & Todos (56) & $\begin{array}{c}172(80) \\
68-390\end{array}$ & $\begin{array}{c}180(86) \\
64-492\end{array}$ & $p=0,703$ \\
\hline $\mathrm{CHDL}^{*}$ & 18 pacientes & $36(11)$ & $41(11)$ & $p=0,067$ \\
\hline$c L D L^{*}$ & 18 pacientes & $142(26)$ & $176(40)$ & $p<0,05$ \\
\hline
\end{tabular}

IAM : infarto agudo de miocardio. CHDL: colesterol de lipoproteínas de alta densidad. CLDL: colesterol de lipoproteínas de baja densidad calculado según fórmula de Friedwald. * Sólo tenían determinación de perfil lipídico completo en ambas situaciones 18 de los 56 pacientes.

éstos fue significativamente menor durante el ingreso $(142 \pm 26$ frente a $176 \pm 40 \mathrm{mg} / \mathrm{dl}, \mathrm{p}<0,05)$. Dos de ellos tenían cLDL entre 100 y 130 y otros 2 menor de 100 mg/dl. Sólo 2 pacientes no cumplieron criterios para iniciar tratamiento hipolipemiante farmacológico en la primera determinación ambulatoria, considerando como tal un cLDL mayor de 130 $\mathrm{mg} / \mathrm{dl}$, pero ambos lo precisaron tras la segunda determinación ambulatoria. Los otros 54 cumplieron ya criterios de tratamiento farmacológico en la primera determinación ambulatoria.

Al dividir la muestra en terciles según la cifra de colesterol medida ambulatoriamente, los del tercil inferior no alcanzaron diferencia significativa con la concentración en fase aguda $(213 \pm 25$ frente a $213 \pm 18 \mathrm{mg} / \mathrm{dl})$, siendo ésta significativa en los dos terciles superiores $(246 \pm 6$ frente a $211 \pm 33, \mathrm{p}<0,01, \mathrm{y}$ $287 \pm 33$ frente a $244 \pm 46 \mathrm{mg} / \mathrm{dl}, \mathrm{p}<0,001)$. Por tanto el mayor descenso en fase aguda se produjo en aquellos con niveles basales más elevados (Fig. 1).

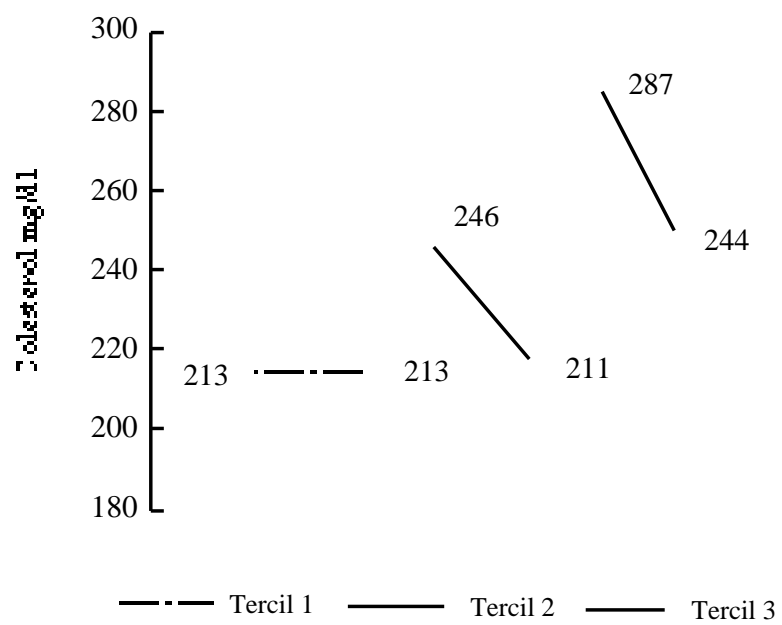

Fig. 1. Descenso en fase aguda según tercil de colesterol en consulta. 


\section{DISCUSIÓN}

La respuesta de fase aguda a la lesión miocárdica tiene un impacto determinante sobre el momento adecuado en que se deben hacer los análisis de lípidos y lipoproteínas. Es importante el conocimiento de los cambios de fase aguda en las concentraciones de lipoproteínas para una adecuada toma de decisiones (3). Comparando con las concentraciones reales basales, un perfil lipoproteíco obtenido más de 24 horas después del comienzo de los síntomas de un infarto agudo de miocardio puede poner de manifiesto concentraciones reducidas de colesterol total, cLDL y cHDL, con elevaciones de triglicéridos y lipoproteína (a) $(3,4)$.

Este trabajo retrospectivo permite confirmar en nuestro centro el descenso de la colesterolemia durante manifestaciones agudas de cardiopatía isquémica en las condiciones del trabajo asistencial diario.

Los conocimientos patogénicos actuales sobre la aterosclerosis y sus manifestaciones clínicas agudas nos permiten afirmar que no sólo hay fenómenos inflamatorios durante el infarto agudo de miocardio sino que, al menos localmente en la placa complicada, éstos se producen también en los cuadros de angina inestable (9). Los cambios lipídicos séricos durante la angina inestable no han sido claramente evaluados. Aunque es minoritario el grupo de pacientes con este diagnóstico en nuestro estudio, esta actividad inflamatoria permite probablemente explicar los cambios en los lípidos en la angina inestable, en el mismo sentido, aunque con menor magnitud, que los ocurridos durante la necrosis miocárdica. La proteína $\mathrm{C}$ reactiva se reconoce en la actualidad como un marcador del proceso inflamatorio asociado a la aterosclerosis (9). Dicha proteína $\mathrm{C}$ reactiva se une selectivamente a las lipoproteínas de muy baja densidad (VLDL) y a las LDL, interfiriendo probablemente con el catabolismo de las VLDL (3). Aunque éste no sea probablemente el único mecanismo patogénico que explique las alteraciones en las concentraciones lipídicas en fase aguda (3), podría permitir explicar también las producidas en el caso de la angina inestable.

Los trabajos que han demostrado el beneficio de las estatinas en prevención secundaria han incluido pacientes que iniciaron su tratamiento al menos 3 ó 6 meses $(1,2)$ después del evento agudo. Los beneficios obtenidos y la descripción de mecanismos de mediación de ese beneficio no claramente dependientes del descenso de la colesterolemia (10) permiten suponer que dichos efectos serían mayores si el tratamiento se iniciara más precozmente tras el episodio agudo. Existen ya evidencias del beneficio sobre algunos de estos mecanismos, como la disfunción endotelial, iniciando el tratamiento en el momento del alta hospitalaria después de ingresos por eventos coronarios agudos (estudio RECIFE con pravastatina en pacientes con colesterolemia superior a $200 \mathrm{mg} / \mathrm{dl}$ en fase aguda) (11). Sin embargo no hay evidencias aún del beneficio de este inicio precoz en cuanto a eventos clínicos; el estudio MIRACL (12) ha randomizado pacientes con angina inestable o IAM sin onda Q dentro de los 4 primeros días de ingreso, siempre que no tuvieran colesterolemia mayor de $270 \mathrm{mg} / \mathrm{dl}$, para recibir $80 \mathrm{mg}$ al día de atorvastatina o placebo, en diseño doble ciego; su objetivo es determinar la utilidad de disminuciones grandes y tempranas del colesterol sérico en síndromes coronarios agudos. Algunas de las últimas reuniones que han debatido este tema para establecer recomendaciones, empiezan a admitir la posibilidad del tratamiento farmacológico más precoz, siempre que exista estabilidad metabólica hepática, se hayan descartado causas secundarias de hipercolesterolemia y no se olvide estimular simultáneamente las medidas higiénico-dietéticas $(13,14)$.

Se confirma también en los presentes datos que los descensos de la colesterolemia son más importantes en aquellos pacientes con cifras de colesterol basales más elevadas, como está recogido en la literatura en las condiciones de observación experimental $(3,4,7)$.

El trabajo presenta varias limitaciones que pueden influir en los resultados recogidos, si bien la mayoría de ellas están también presentes en los estudios de observación prospectivos que describieron el conocido descenso de la colesterolemia en fase aguda. No está recogida la eficacia e influencia de las medidas higiénico-dietéticas emprendidas al alta hospitalaria, ni la posible influencia de los cambios ponderales. Sin embargo, al ser una fase en que los pacientes están especialmente estimulados para el cambio de hábitos, lo esperable es un descenso de peso. La posible influencia de éste reforzaría en todo caso los hallazgos; si no se hubiera producido el descenso de peso la colesterolemia, aunque sin una relación tan directa con el peso como los triglicéridos, sería probablemente más elevada. Tampoco se recogieron la utilización de trombolíticos ni los fármacos que fueron indicados al alta hospitalaria, excepto la confirmación de la ausencia de tratamiento hipolipemiante farmacológico. Algunos de los fármacos más habitualmente utilizados pueden modificar las concentraciones lipídicas (betabloqueantes, diuréticos). La trombolisis no está claro si acrecienta o no estos cambios (3), si bien hay algún trabajo que apoya un mayor descenso en fase aguda de las concentraciones de colesterol si se realiza trombolisis.

Con las limitaciones expuestas, y basándonos tanto en los presentes datos como en la revisión de la literatura, conside ramos que se puede sugerir:

1. Es recomendable la obtención precoz, antes de las 24 horas desde el inicio de los síntomas del evento agudo, de un perfil lipídico, incluyendo cHDL que permita el cálculo de cLDL. Sin embargo la falta de ayuno pre-analítico en este tipo de extracciones puede influir en el cálculo del cLDL y por tanto en una clasificación terapéutica adecuada.

2. Se deben buscar en la historia del paciente determinaciones lipídicas previas que nos permitan una adecuada clasificación. Si en algún paciente es difícil justificar que no reciba tratamiento hipolipemiante precoz después de un evento coronario es en aquel que ya tenía concentraciones de colesterol elevadas previamente. El estudio PREVESE-98 sigue poniendo en evidencia un tratamiento hipolipemiante deficitario postinfarto incluso en este grupo de pacientes en nuestro sistema sanitario (15).

3. Los descensos de la concentración de colesterol que se producen en fase aguda son de magnitud suficiente para que, aún con una buena adherencia a las modificaciones en los hábitos higiénico-dietéticos, no se mantenga dicho descenso. Este hecho permitiría, en caso de no disponer de determinaciones lipídicas por alguna de las dos opciones previas, que es la situación representada por la muestra de nuestro estudio, decidir el inicio de tratamiento farmacológico basándose en la única determinación del ingreso, sin transgredir las recomendaciones actuales. Al menos se debería cumplir lo que indica el reciente documento de consenso para el control de la colesterolemia en España (16): "Las personas con elevaciones importantes del cLDL y del colesterol total, superiores a 160 y 
$240 \mathrm{mg} / \mathrm{dl}$, respectivamente, deben recibir tratamiento farmacológico durante el ingreso hospitalario".

4. En pacientes en que se decida posponer el inicio del tratamiento farmacológico hipolipemiante es importante la repetición precoz de las determinaciones, en 4 a 6 semanas, para reevaluar la decisión sobre dicho tratamiento y no privarles de un tratamiento con beneficio demostrado.
Sin pretender generalizar a partir de estos datos ninguna recomendación, su difusión sí pretende estimular la recogida de experiencias similares que, junto a los futuros datos de estudios con intervención hipolipemiante más precoz, permitan sustentar en evidencias la recomendación de inicio más temprano del tratamiento con estatinas después de un evento coronario agudo.

\section{Bibliografía}

1. Sacks FM, Pfeffer MA, Moye LA, Rouleau JL, Rutherford JD, Cole TG, et al, for the Cholesterol and Recurrent Events Trial Investigators. The effect of pravastatin on coronary events after myocardial infarction in patients with average cholesterol levels. $\mathrm{N}$ Engl $\mathrm{J}$ Med 1996;335:1001-1009.

2. Scandinavian Simvastatin Survival Study Group. Randomised trial of cholesterol lowering in 4.444 patients with coronary heart disease: the Scandinavian Simvastatin Survival Study (4S). Lancet 1994;344:1383-1389.

3. Rosenson RS. Myocardial injury: the acute phase response and lipoprotein metabolism. J Am Coll Cardiol 1993;22:933-40.

4. Cooper GR, Myers GL, Smith SJ, Schalant RC. Blood lipid measurements. Variations and practical utility. JAMA 1992;267:1652-1660.

5. Tibblin G, Cramer K. Serum lipids during the course of an acute myocardial infarction and one year afterwards. Acta Med Scand 1963;174:451-5.

6. Ryder RE, Hayes TM, Mulligan IP, Kingswood JC, Williams S, Owens DR. How soon after myocardial infarction should plasma lipid values be assessed? Br Med J 1984;289:1651-3.

7. Gore JM, Goldberg RJ, Matsumoto AS, Castelli WP, McNamara PM, Dalen JE. Validity of serum total cholesterol level obtained within 24 hours of acute myocardial infarction. Am J Cardiol 1984;54:722-5.

8. Prevention of coronary heart disease: scientific background and new clinical guidelines. Recommendations Of the European Atherosclerosis Society prepared by the International Task Force or Prevention of Coronary Heart Disease. Nutr Metab Cardiovasc Dis 1992;2:113-156.

9. Ross R. Atherosclerosis - An inflammatory disease. N Engl J Med 1999;340:115-126.

10. Rosenson RS, Tangney CC. Antiatherothrombotic properties of statins.

Implications for cardiovascular event reduction. JAMA 1998;279:16431650.

11. Dupuis J, Tardif JC, Cernacek P, Théroux P. Cholesterolreduction repidly improves endothelial function after acute coronary syndromes. The RECIFE (Reduction of Cholesterol in Ischemia and Function of the Endothelium) trial. Circulation 1999;99:3227-33.

12. Schwartz GG, Oliver MF, Ezekowitz MD, Ganz P, Waters D, Kane JP et al. Ratinale and design of the myocardial ischemia reduction with aggressive cholesterol lowering (MIRACL) study that evaluates Atorvastatin in unstable angina pectoris end in non-Q-wave acute myocardial infarction. Am J Cardiol 1998;81:578-81.

13. Grundy SM, Balady GJ, Criqui MH, Fletcher G, Greenland P, Hiratzka $\mathrm{LF}$, et al. When to start cholesterol-lowering therapy in patients with coronary heart disease. A statement for healthcare professionals from the American Heart Association Task Force on Risk Reduction. Circulation 1997;95:1683-85.

14. Wood D, De Backer G, Faergeman O, Graham I, Mancia G, Pyörälä K, et al. Prevention of coronary heart disease in clinical practice. Recommendations of the Second Joint Task Force of European and other Societies on Coronary Prevention. Eur Heart J 1998;19:1434-1503.

15. De Oya M, López Sendón JL, de Teresa E, Velasco-Rami J, Villa E, Cosín J. The impact of landmark clinical trials on secondary prevention of acute myocardial infarction (AMI) in Spain. Prevese 98 study. Atherosclerosis 2000;151:107.

16. Ministerio de Sanidad y Consumo, Sociedad Española de Cardiología y Sociedad Española de Arteriosclerosis. Control de la colesterolemia en España, 2000. Un instrumento para la prevención cardiovascular. Clin Invest Arteriosclerosis 2000;12:125-152. 